\title{
Controlling uphill motion of an active Brownian particle driven by shot-noise energy pulses
}

\author{
Alessandro Fiasconaro, ${ }^{1, *}$ Ewa Gudowska-Nowak, ${ }^{2,3, \dagger}$ and Werner Ebeling ${ }^{2,4, \ddagger}$ \\ ${ }^{1}$ Departamento de Física de la Materia Condensada and ICMA (CSIC-Universidad de Zaragoza), 50009 Zaragoza, Spain \\ ${ }^{2}$ Mark Kac Complex Systems Research Center, Jagiellonian University, Reymonta 4, 30-059 Kraków, Poland \\ ${ }^{3}$ Marian Smoluchowski Institute of Physics, Jagiellonian University, Reymonta 4, 30-059 Kraków, Poland \\ ${ }^{4}$ Institute of Physics, Humboldt University Berlin, Newtonstr. 15, 12489 Berlin, Germany
}

(Received 6 November 2012; published 6 March 2013)

\begin{abstract}
We study self-propelled motion of an active Brownian particle moving in a periodic, ratchet-type potential and subject to energy support distributed in quantized portions according to a Poisson spiking process. The motor features of such a system are examined by analyzing its ability to perform work against additional external load. The control parameter of the system is a mean duration time between subsequent energy pulses. Our analysis indicates that directionality of the motion depends strongly on the correlation time between events of energy supply and can be adjusted to maintain optimal functionality of the motor.
\end{abstract}

DOI: 10.1103/PhysRevE.87.032111

PACS number(s): 05.40.-a, 05.45.-a

\section{INTRODUCTION}

Unlike passive Brownian particles whose drifting movement is triggered by random collisions with light particles of the environment, motion of active "brownons" is set going by consumption of energy pumped into the system. A combined action of Brownian random forces and of the energy-powered propulsion mechanism is by now a well established model for motility of cells or microorganisms [1-5] and functioning of molecular biodevices [6-9]. Many molecular catalysts, motors, or pumps convert chemical energy into motion which can be illustrated as a (biased) random walk on a representative free energy landscape. Models of passive long molecules translocation driven by motors have been also recently studied [10-13]. Collective effects of such groups of motors have been also addressed and existing literature points to a possible mapping of the stochastic dynamics of coupled molecular motors to models of active Brownian motion (ABM) $[1,14,15]$.

The main objective of this work is to investigate a concerted influence of thermal noise and random energy uptake on the performance of a ratchet system working within the framework of ABM [16-21]. The Langevin dynamics for such systems involves the friction coefficient which depends nonlinearly on the particle speed, thus reflecting the way the energy which flows into the system transfers to the dynamic degrees of freedom and dissipates to the medium. Under nonequilibrium conditions, this velocity-dependent friction may attain negative values at low particle speed indicating an uptake of energy from external sources. At large speed the friction becomes positive indicating the dissipation of mechanical energy of motion. Accordingly, the dynamics of the ABM system does not follow fluctuation relations typical for thermodynamic equilibrium (the Einstein relation can be violated) and therefore represents inherently nonequilibrium setups.

The movement and direction of a test particle is studied here in a ratchet potential, under the presence of an additional

\footnotetext{
*afiascon@unizar.es

†gudowska@th.if.uj.edu.pl

¥ebeling@physik.hu-berlin.de
}

constant load force $[19,20,22-24]$ and subject to weak thermal fluctuations. The system is powered by external energy pulses which are supplied in the form of a shot noise with exponentially distributed waiting times. The absorption of energy quanta described as a series of Poisson-distributed events contributes to the energy accumulated in the depot (reservoir). In the next step, some portion of the energy from the depot becomes transduced into work.

Similar ABM dynamics has been previously explored in a piecewise-linear ratchet potential $[19,20]$. Further studies of the directionality of such motion under the action of a smooth (sinusoidal) ratchet-type potential have detected interesting features of the speed saturation and velocity inversion as a function of the energy transfer between the energy depot and the mechanical dynamics [22]. For specific, well adjusted initial conditions, the motion against a load force has been shown [23] to display a quasideterministic uphill movement, i.e., motion against a gradient of the potential and opposing the direction of the load force. Furthermore, depending on the intensity of thermal noise, the system exhibits a change of directionality of motion with the flux of particles going against or in accord with the slope of the potential [23,24].

In this numerical study we concentrate on three physical issues: We discuss the directionality of the motion by measuring a fraction of trajectories performing the uphill motion among all simulated ones under the same conditions, the mean velocity of the active Brownian particle, and the efficiency of energy transformation in the system.

A standard ratchet system can rectify thermal fluctuations and as a consequence direct a current of particles against the load force. For a stopping force (too heavy load), the absolute value of the current vanishes and the device stops operating $[25,26]$. In other words, the ratchet system works as a motor only within the region where the current opposes the load force $F_{0}$ and moving particles perform some work. Accordingly, as a measure of the efficiency we introduce a parameter which compares the power of the motor (or the energy produced in the course of motion per unit time) with respect to the input of energy per unit of time:

$$
\eta_{F_{0}}=\frac{\left|F_{0}\langle v\rangle\right|}{\langle q(t)\rangle} .
$$


We will show here how the above mentioned quantities depend on the environmental noise strength, and more specifically, on the timing of the energy absorption. As it will be demonstrated in the forthcoming sections, the presence of a positive maximum in the asymptotic mean velocity, as well as in the corresponding right trajectory statistics and efficiency $\eta$, indicates that the modeled motor system is able to perform work against the load force once the proper mean time between the energy shots is chosen.

\section{MODEL}

Dynamics of energy transfer between the motor and the depot takes into account variations of energy in the depot system. The equation for the balance of energy involves the source term and the rate of energy loss. In the most general form it can be described by the stochastic differential equation

$$
\frac{d e}{d t}=q(t)-\int_{0}^{t} d t^{\prime} \varphi\left(t-t^{\prime}\right) e\left(t^{\prime}\right)
$$

which incorporates an energy inflow $q(t)$ modeled as a shotnoise process and dissipation with a certain memory kernel $\varphi\left(t-t^{\prime}\right)$. In a first approximation we will assume that the energy input $q(t)$ results from the accumulation of energy "quanta" arriving at the depot as a train of (sharp) impulses:

$$
q(t)=\sum_{i}^{n(t)} Q \delta\left(t-t_{i}\right)
$$

Here $Q$ stands for a constant energy of a single spike, and $t_{i}$ represents the arrival times which are random events from a homogeneous Poisson point process [27-30]. Accordingly, the number of energy quanta $n(t)$ accumulated up to time $t$ follows the Poisson statistics

$$
P(n(t)) \equiv \operatorname{Prob}[n(t)=n]=\frac{t^{n}}{\tau_{c}^{n} n !} \exp \left(-\frac{t}{\tau_{c}}\right),
$$

where $\tau_{c}$ defines the average time between two pulses. We assume, therefore, that the system absorbs energy in packets (quanta) of about the same intensity $Q$ with the impulse response given by a Dirac $\delta$ function.

Consequently the only free parameters are connected with the distribution of the discrete times and in particular with the average time $\tau_{c}=\left\langle t_{i+1}-t_{i}\right\rangle$ between the arrival times of energy shots $Q$. Note that this mechanism mimics the act of absorption of subsequent adenosine triphosphate (ATP) molecules during the mean time $\tau_{c}$, which depends on concentration, steric factors, etc. We also notice that the time $\tau_{c}$ is inversely proportional to the average energy flux per unit time [see Eq. (8) below], which is the mean energy consumption of the device, one of the most important characteristics of the motors.

As a particular model for the energy transfer between the depot and the motor we choose the Schweitzer-EbelingTilch (SET) variant [19-21] which assumes conversion of the internal energy into a kinetic energy of motion with a momentum dependent rate $d v^{2}$. Moreover, the internal energy dissipates with the constant rate, so that the energy balance
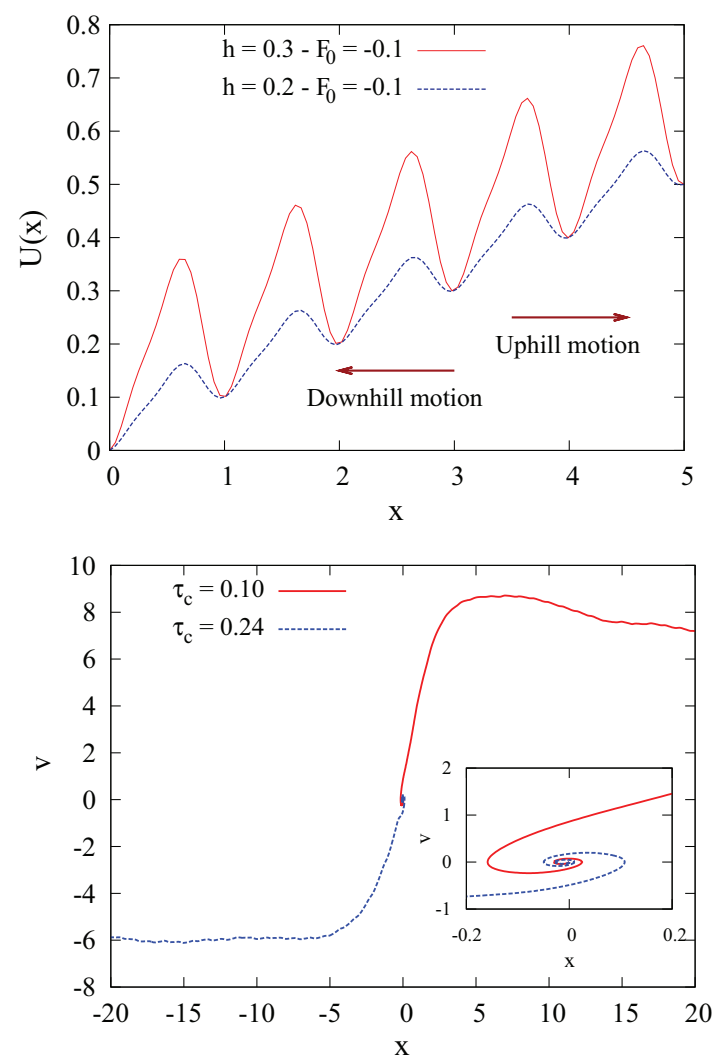

FIG. 1. (Color online) (Upper panel) Ratchet potential as used for simulations for two different values of the height $h=0.2,0.3$. Parameter values are $a=0.499, b=0.453$, and $\phi=0.1903$. The external load $F_{0}=-0.1$ is modeled as an additional bias pointing to the left. (Lower panel) Examples of an uphill trajectory [with positive velocity $v(t)>0$ ] for $\tau_{c}=0.1$ and a downhill trajectory [with negative velocities $v(t)<0$ ] for $\tau_{c}=0.24$, both calculated for $h=0.28, c=0.1$, and $d=0.1$. Inset of the lower panel: zoom of the central part of the figure illustrating a transient behavior during the exit out of the potential well.

equation reads

$$
\frac{d e(t)}{d t}=\sum_{i} Q \delta\left(t-t_{i}\right)-c e(t)-d v^{2}(t) e(t) .
$$

By assuming a unitary mass of the Brownian particle, the corresponding equation of motion takes the form of $[20,22]$

$$
\frac{d v(t)}{d t}+\gamma_{0} v(t)=F(x)+d e(t) v(t)+\sqrt{2 D} \xi(t),
$$

where the total force $F(x)=F_{0}-U^{\prime}(x)$ with the spatially periodic potential given by superposition [31]:

$$
\frac{U(x)}{h}=a-b\left\{\sin [2 \pi(x+\phi)]+\frac{1}{4} \sin [4 \pi(x+\phi)]\right\} .
$$

Here the spatial period of the potential $U(x)$ is set to $l_{0}=1$ and $h$ stands for the height of the barrier between subsequent potential minima (Fig. 1). The force $\xi(t)$ in Eq. (6) stands for external random perturbances, statistically independent of energy variations. We model $\xi(t)$ by the Gaussian white noise whose intensity is scaled with $D$. 
The total mean energy $\langle q(t)\rangle$ delivered to the depot system changes with the mean number of spikes $\langle n(t)\rangle \equiv t / \tau_{c}$, that is,

$$
\langle q(t)\rangle=Q\langle n(t)\rangle=Q \frac{t}{\tau_{c}} .
$$

The mean time $\tau_{c}$ between the energy spikes $Q$ is here considered a free, tuning parameter. With respect to molecular motors the quantity $n(t)$ can be related to the number of ATP molecules bond to the machine in time interval $t$. We will further assume as energy unit $Q$ the energy released by an ATP molecule in living cells, say $E_{A T P} \simeq 15 k_{B} T$ to $20 k_{B} T$ [6]. With the parameters used in our analysis, the height of the barrier $h=0.28$ corresponds in units of $Q$ to the energy of about $4.2 k_{B} T$ to $5.6 k_{B} T$, a value very close to other estimations of a typical barrier height of a biological ratchet device [9].

It is worth to note that energy $e(t)$ contributes into the particle dynamics as a multiplicative noise which couples to the variable $v(t)$; cf. Eq. (6). This stochastic energy contribution (similar to its equivalent model studied in [24] and to the deterministic energy supply model analyzed in [19-23]) breaks the time-reversal symmetry of the system which, according to the paradigm of a ratchet mechanism, is a fundamental requirement for a net displacement. The multiplicative noise (either stochastic or deterministic) affects the dynamics by confining the system to a subspace of the phase space, with either left or right velocity. In turn, the presence of another, independent additive noise term tends to restore the symmetry of the dynamics by mixing trajectories of the two directionality states (See also Refs. [5,32]).

\section{RESULTS}

As we have shown earlier [22,23], the ratchet system driven by active friction with a strong depot-particle coupling possesses two momentum-dependent attractors. In the stochastic description we have correspondingly two classes of trajectories: the uphill trajectories, corresponding to the active motor-regime with positive velocity opposite to the direction of a bias (load force $F_{0}$ ), and the downhill ones, with negative velocity, which correspond to an idle motor run. If, after the transient state, the system sets in the motor regime, it stays there and performs work against the bias, provided that the external noise $\xi(t)$ is not too strong. With stochastic driving force $\xi(t)$ in Eq. (5), in the limit of $\dot{e}=0$, the stationary velocity distribution function $P_{s t}(v)$ of the model becomes bimodal and for $F_{0}=U^{\prime}(x)=0$, its maxima are located symmetrically around $v=0$. They correspond to two (left or right) directed stochastic fluxes. A suitable choice of parameters and initial conditions in the noise-free case $(D=0)$ yields one of the two fluxes, so that the overall current of particles becomes practically unidirectional, moving to the left or to the right as a function of the initial conditions [22]. The direction of motion is determined by the sign of the velocity achieved by the particle during the transient period when its kinetic energy reaches the level ample to exit from the potential well. Unlike in formerly investigated cases [22-24], the motor model analyzed here does not give a constant asymptotic value of the velocity at a high rate of input energy shots or, alternatively, at very short waiting times between

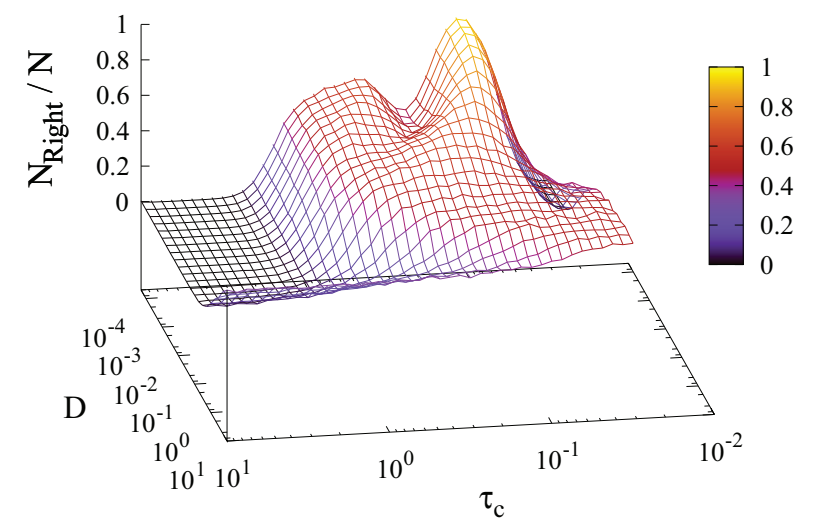

FIG. 2. (Color online) Fraction of right trajectories as a function of the noise intensity $D$ and the mean waiting time between energy spikes $\tau_{c}$. Simulations have been run with initial conditions $x(0)=$ $0, v(0) \sim 0$.

subsequent energy supplies (see Fig. 3). In other words, at a high rate, when the energy supplied tends to infinity, so also does the modulus of the velocity. Moreover the system stops the uphill motion, presenting a negative velocity. This indicates that there exists a maximum delivery rate at which the motor can actually work actively against the load. On the other hand this limit is physically natural, because of the existence of a natural cut-off, given by the hydrolyzation time of the ATP molecules, which gives the minimum working time of the machine, and consequently a minimum time between subsequent bond and usage of energy quanta. The maximum of the efficiency function analyzed in terms of the delivery time $\tau_{c}$ corresponds to the optimal rate of energy absorption.

In the case of shot noise only (without the thermal one), the energy inflow $q(t)$ does not change qualitatively the dynamic scenario and via numerical simulations we can detected relatively stable, nonzero particle currents directing to the right [22-24]. In turn, the presence of both noise terms mixes preferential initial conditions in such a way that the left or right fluxes cannot be established in advance due to the fluctuating behavior of the velocity during the transient.

For sufficiently long times and with increasing values of the noise intensity, the system is able to make transitions between the groups of trajectories pointing to the left or right, thus reflecting a sensibility of boundaries between the two attractors to stochastic effects [23]. For low noise intensities, the transitions between different directions of motion are quite seldom, and the motion stabilizes at the velocity achieved during the initial transient state. Figure 2 shows the overall strong dependence of the right directed flux from both the thermal noise intensity $D$ and the waiting times between energy spikes $\tau_{c}$, and shows how relevant can be the role played by the two parameters in the active motion. Of particular importance in this context is the presence of a very clear maximum in a region of $D-\tau_{c}$ space, which delineates the regime of parameters for which the uphill motion occurs. A selection of exemplary curves (projections of Fig. 2) has been plotted in Fig. 3 for noise intensities $D=10^{-4}, 10^{-3}, 10^{-2}, 10^{-1}$. Additionally, the upper panel of Fig. 3 shows the mean velocity of the particles $\langle v\rangle$ estimated according to the following definition in the long 

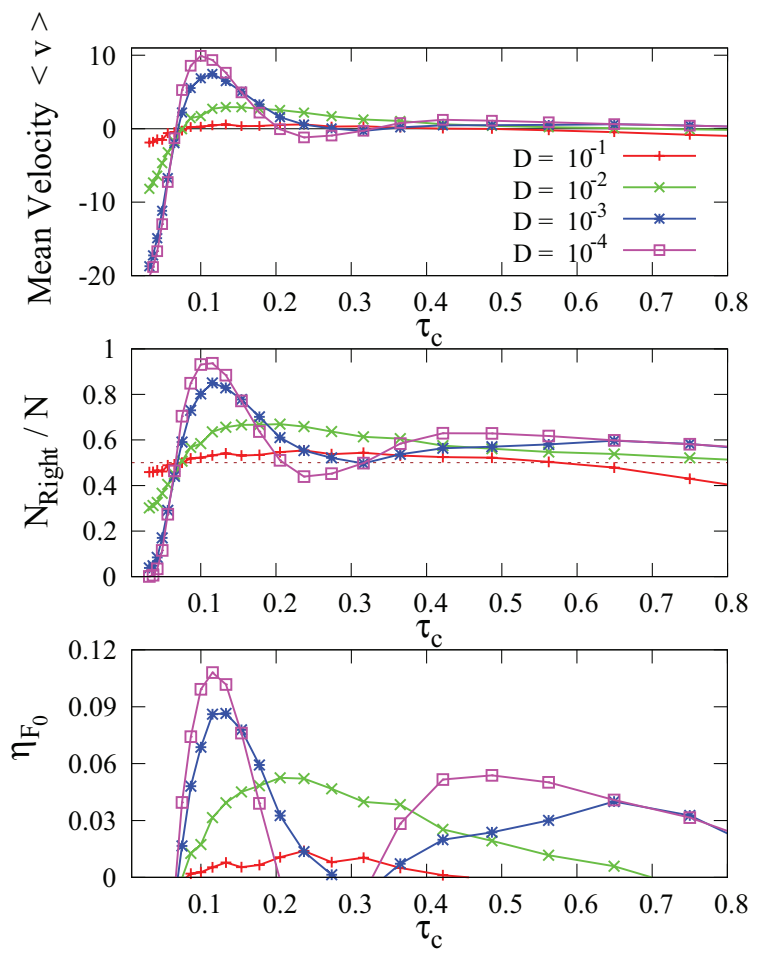

FIG. 3. (Color online) (Upper two panels) Mean velocity of the particles and fraction of the right trajectories as a function of the mean waiting time $\tau_{c}$. The behavior appears nonmonotonic, indicating that the timing of energy spikes affects the directionality of the flux of particle. (Lower panel) The efficiency $\eta_{F_{0}}$ of conversion of input energy into the uphill work of the motor. The strength of a white noise is given as a parameter.

time approximation:

$$
\langle v\rangle=\frac{1}{N} \sum_{i=1}^{N} \frac{x_{i}\left(T_{\max }\right)}{T_{\max }} .
$$

Here $x_{i}\left(T_{\max }\right)$ is the position reached by the test particle at the maximum simulation time $T_{\max }$. ( $T_{\max }=300$ has been preset in all numerical experiments performed with a time step $d t=$ $10^{-3}$. The number of independent simulations $N=5000$.)

The average velocity of motion [Eq. (9)] and the fraction of the uphill trajectories demonstrate a maximum for $\tau_{c} \simeq 0.1$. This resonant value of the flux corresponds to about $93 \%$ of trajectories following the uphill direction indicating a run of the motor working against the load. Furthermore, the lower panel of Fig. 3 shows the efficiency of the motor, according to the definition given in Eq. (1), which reproduces qualitatively the behavior depicted in both $\langle v\rangle$ and $N_{\text {Right }} / N$.

The highest value of the efficiency is about $10 \%$, a value slightly smaller than the one calculated recently for stepper motors [33]. Again, the optimal motor regime with respect to efficiency is reached if the mean waiting time between the shots is around $\tau_{c} \simeq 0.1$ time units, where the maximum in the right oriented trajectories and the maximum of the mean velocity are recovered.

As can be inferred from Fig. 4 which displays the mean velocity as a function of the noise intensity $D$, at low values of external noise $\left(D \lesssim 10^{-4}\right)$ the flux preserves a well defined direction, because the accidental stochastic force is too weak to

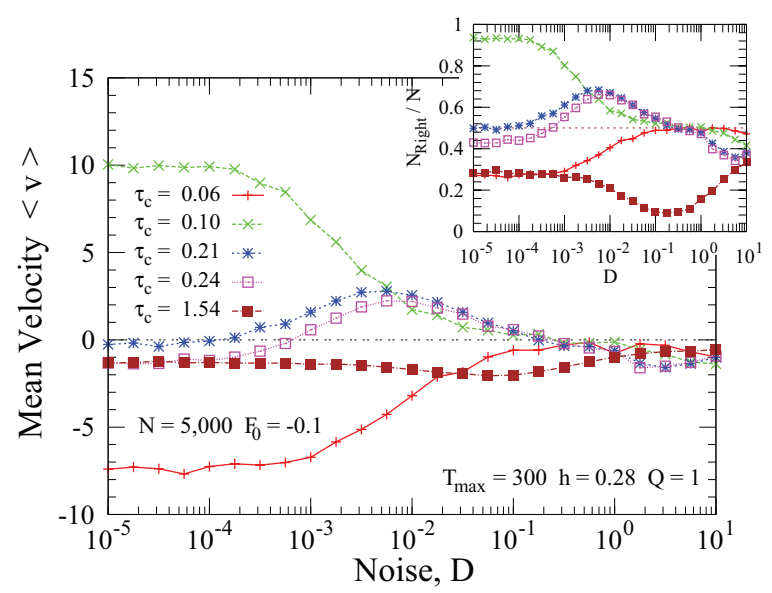

FIG. 4. (Color online) Mean velocity as a function of the white noise intensity $D$ for different mean waiting times $\tau_{c}$. The inset shows the corresponding fraction of right trajectories.

cause change in the overall orientation of motion. In contrast, at strong noise mostly negative fluxes (downhill motion) are observed.

For $\tau_{c}=0.21$ to 0.24 the motion at low $D$ is composed only in $40 \%$ to $50 \%$ of right pointing trajectories. The inversion of motion towards positive velocities can be, however, induced by increasing intensity $D$ of the additive noise (cf. inset of Fig. 4).

Examples of motion with the two possible directions are further reported in Fig. 5 for $D=10^{-4}$, where the mean velocity (upper panels) and the corresponding energy of the depot (lower panels) are shown for two waiting time values $\tau_{c}=0.1$ and $\tau_{c}=0.24$, which correspond to the resonant positive and to the resonant negative values of the velocity, respectively.
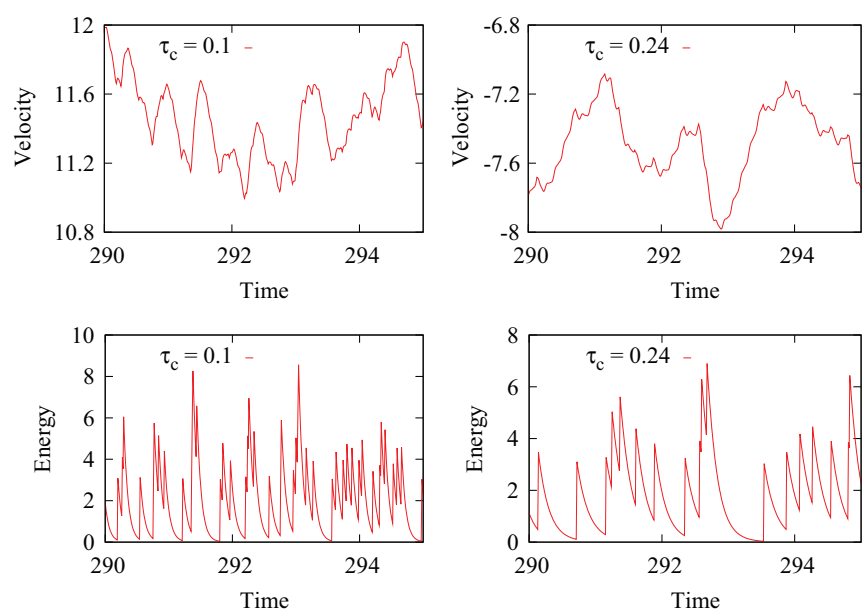

FIG. 5. (Color online) Examples of time variations of velocity and energy in the system for the weak noise intensity $D=10^{-4}$ and different waiting times between energy spikes. Left and right panels demonstrate variations in uphill and downhill trajectories, respectively. The $\tau_{c}$ values correspond to the first resonant maxima visible in Fig. $3\left(\tau_{c}=0.1\right)$ and to a value at which negative velocities become preferential $\left(\tau_{c}=0.24\right)$. 

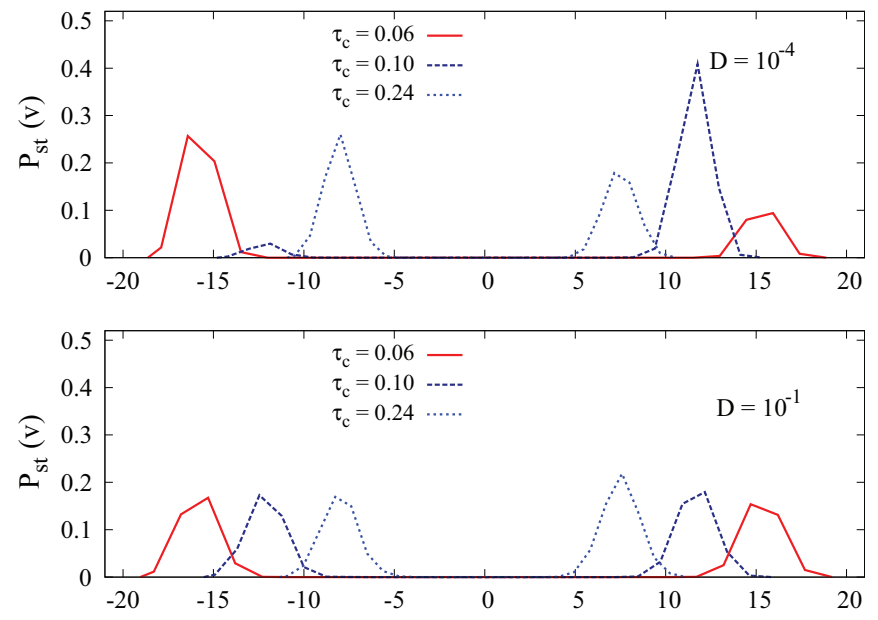

FIG. 6. (Color online) Bimodal stationary velocity distribution for different waiting times $\tau_{c}$ and two noise intensities $D$, namely, $\tau_{c}=0.06,0.1,0.24, D=10^{-4}$, and $D=10^{-1}$. The right peak which corresponds to the uphill motion against the load is separated from the left peak by a valley of less probable states (regime of unstable velocities). Statistical analysis has been performed on a set of $N$ numerical experiments $(N=5000)$ with the simulation time $T_{\max }=$ 300. The simulation time step has been set to $d t=10^{-3}$.

In order to better clarify the directionality features of the current, we have analyzed the long time (stationary) velocity probability distribution function $P_{s t}(v)$, estimated from the trajectories $x_{i}\left(T_{\max }\right)$ (see Fig. 6). The mean velocities $\langle v\rangle$ have been evaluated according to the definition Eq. (9) and compared with the first moment of $P_{s t}(v)$ showing perfect agreement with the values plotted in Fig. 3 and in Fig. 4. This observation confirms that the evaluation times as used in our numerical studies are long enough to guarantee proper thermalization of the system. Figure 6 shows how strong and clear is the change of direction for $D=10^{-4}$ (upper panel with $\tau_{c}=0.06$, negative, to $\tau_{c}=0.10$, positive, and again $\tau_{c}=0.24$, negative), while for $D=10^{-1}$ the same qualitative behavior appears much less evident (lower panel).

The most important property demonstrated in Fig. 6 is the bimodality of the velocity probability density, which is related to the properties of the multiplicative noise [5,32] entering into the dynamics [Eq. (6)] via the coupling term $d v(t) e(t)$. As a result, two possible modes of uphill and downhill motion are well separated by a relatively long interval of unprobable (metastable) dynamic states. Under the conditions investigated the transitions between the two modes occur quite seldom at low values of the additive noise intensity $D$. In consequence, we conclude that in the case when the system is brought by initial conditions into the transitory uphill mode, it can stay there for a quite long time before it switches to the opposite, downhill motion.
In other words, in the bistable stochastic state, the motor regime (giving positive velocities) is a metastable state, which however presents here a very long working time. Only after that time, the motor will need new preparation, i.e., a new setting up of the initial conditions to the motor regime.

\section{CONCLUSIONS}

Standard models of molecular motors are based on the Smoluchowski equations [7,8] for discrete systems having several states which correspond to attachment or detachment of the motor from a molecular track. Many models have been developed which follow similar lines [1,5,34,35]. We have investigated the motion of a particle against a gradient of the potential, i.e., uphill motion under conditions where the external force is constant and directed to the left. A fairly general schema is that the energy is absorbed and introduced into our minimalistic "machine" by increasing the energy of the reservoir by a certain amount. The energy support is modeled here as a discrete inflow of energy quanta (ATP shots) arriving with an exponential distribution of waiting times. The energy inflow to the motor becomes further transformed into mechanical energy of motion. We have shown that, for the particular model studied in this paper, a suitable choice of kinetic (and ratchet) parameters allows one to induce an uphill motion of particles by tuning both the mean time between subsequent energy shots and the intensity of the external Gaussian noise. The stationary velocity distribution function of the model is in general bimodal, demonstrating peaks of probability in both the uphill regime (active motor regime) and in the downhill regime (idle run of the motor). This indicates that the uphill run of our stochastic motor is rather an intermittent phenomenon and requires specific conditions (weak external noise and tuning of the delivery time of energy quanta) to stabilize the motion.

Performance of the motor can be addressed by studying efficiency and analyzing statistics of sample paths (trajectories) in a demanded direction. For the model system analyzed in the paper the efficiency and the fraction of uphill trajectories demonstrate a strong dependence on the mean waiting time between the energy shots. This observation suggests that the mean waiting time between the arrival of energy quanta (mimicking, e.g., the acts of ATP absorption) is very likely one of the most important parameters for optimization of biological machines.

\section{ACKNOWLEDGMENTS}

This work was partially supported by Spanish MICINN through DGICYT Project No. FIS2011-25167, and cofinanced by Fondo Europeo de Desarrollo Regional (FEDER) funds and by the European Science Foundation/EPSD (Exploring Physics of Small Devices) project. E.G.-N. acknowledges Grant No. MPD/2009/6 from the Foundation for Polish Science. The authors want also to thank Dr. Fernando Falo for useful discussions.
[1] B. Lindner and E. M. Nicola, Eur. Phys. J. Special Topics $\mathbf{1 5 7}$ 43 (2008).
[2] J. Strefler, W. Ebeling, E. Gudowska-Nowak, and L. Schimansky-Geier, Eur. Phys. J. B 72, 597 (2009). 
[3] D. Selmeczi, S. Mosler, P. H. Hagedorn, N. B. Larsen, and H. Flyvbjerg, Biophys. J. 89, 912 (2005).

[4] D. Selmeczi, L. Li, L. I. I. Pedersen, S. F. Nrrelykke, P. H. Hagedorn, S. Mosler, N. B. Larsen, E. C. Cox, and H. Flyvbjerg, Eur. Phys. J. Special Topics 157, 115 (2008).

[5] P. Romanczuk, M. Baer, W. Ebeling, B. Lindner, and L. Schimansky-Geier, Eur. Phys. J. Special Topics 202, 1 (2012).

[6] P. Nelson, Biological Physics: Energy, Information, Life (W. H. Freeman and Company, New York, 2004).

[7] F. Jülicher and J. Prost, Phys. Rev. Lett. 75, 2618 (1995).

[8] F. Jülicher, A. Ajdari, and J. Prost, Rev. Mod. Phys. 69, 1269 (1997).

[9] L. Machura, M. Kostur, and J. Luczka, BioSystems 94, 253 (2008).

[10] A. Fiasconaro, J. J. Mazo, and F. Falo, Phys. Rev. E 82, 031803 (2010).

[11] J. A. Cohen, A. Chaudhuri, and R. Golestanian, Phys. Rev. Lett. 107, 238102 (2011).

[12] A. Fiasconaro, J. J. Mazo, and F. Falo, J. Stat. Mech. (2011) P11002.

[13] A. Fiasconaro, J. J. Mazo, and F. Falo, New J. Phys. 14, 023004 (2012).

[14] C. A. Condat and M. E. Di Salvo, Phys. Rev. E 84, 011911 (2011).

[15] C. Touya, T. Schwalger, and B. Lindner, Phys. Rev. E 83, 051913 (2011).

[16] W. Ebeling and I. Sokolov, Statistical Thermodynamics and Stochastic Theory of Nonequilibrium Systems (World Scientific, Singapore, 2005).

[17] F. Schweitzer, Brownian Agents and Active Particles, Springer Series in Synergetics (Springer, Berlin, 2003).
[18] A. S. Mikhailov and V. Calenbuhr, From Cells to Societies, Springer Series in Synergetics (Springer, Berlin, 2002).

[19] W. Ebeling, F. Schweitzer, and B. Tilch, BioSystems 49, 17 (1999).

[20] F. Schweitzer, W. Ebeling, and B. Tilch, Phys. Rev. Lett. 80, 5044 (1998).

[21] U. Erdmann, W. Ebeling, L. Schimansky-Geier, and F. Schweitzer, Eur. Phys. J. B 15, 105 (2000).

[22] W. Ebeling, E. Gudowska-Nowak, and A. Fiasconaro, Acta Phys. Pol. B 39, 1251 (2008) [http://th-www.if.uj.edu.pl/acta/ vol39/pdf/v39p1251.pdf].

[23] A. Fiasconaro, W. Ebeling, and E. Gudowska-Nowak, Eur. Phys. J. B 65, 403 (2008).

[24] A. Fiasconaro, E. Gudowska-Nowak, and W. Ebeling, J. Stat. Mech. (2009) P01029.

[25] J. M. R. Parrondo and B. J. De Cisneros, Appl. Phys. A 75, 179 (2002).

[26] L. Machura, M. Kostur, P. Talkner, J. Łuczka, and P. Hänggi, Phys. Rev. Lett 98, 040601 (2007).

[27] R. Zygadlo, Phys. Rev. E 47, 4067 (1993).

[28] T. Czernik and J. Łuczka, Ann. Phys. 9, 721 (2000).

[29] I. Eliazar and J. Klafter, Proc. Natl. Acad. Sci. USA 27, 13779 (2005).

[30] C. Kim, E. K. Lee, P. Hänggi, and P. Talkner, Phys. Rev. E 76, 011109 (2007).

[31] J. L. Mateos, Phys. Rev. Lett. 84, 258 (2000).

[32] F. de Pasquale, J. M. Sancho, M. San Miguel, and P. Tartaglia, Phys. Rev. A 33, 4360 (1986).

[33] M. Zabicki, W. Ebeling, and E. Gudowska-Nowak, Chem. Phys. 375, 472 (2010).

[34] M. M. Millonas and M. I. Dykman, Phys. Lett. A 185, 65 (1994).

[35] F. Takagi and T. Hondou, Phys. Rev. E 60, 4954 (1999). 